\title{
Functional Connection Changes among Cerebral Regions and between Hemispheres in the Acute Ischemic Stroke Patients Treated by Scalp Acupuncture: A Rs-fMRI Study
}

Huacong Liu

Southern Medical University

Yijing Jiang

Fujian University of Traditional Chinese Medicine

Ningning Wang

Community Health Services of Houjie Town

\section{Lanpin Chen}

Southern Medical University

Jingchun Gao

Third Affiliated Hospital of Southern Medical University

Jiping Zhang

Southern Medical University

Shanshan Qu

Southern Medical University

Songyan Liu

China-Japan Union Hospital of Jilin University

\section{Gang Liu}

Third Affiliated Hospital of Southern Medical College

\section{Yong Huang}

Southern Medical University

Junqi Chen ( $\sim$ meixibao@126.com )

Third Affiliated Hospital of Southern Medical University https://orcid.org/0000-0001-6847-0891

\section{Research}

Keywords: the Resting State Functional Magnetic Resonance Imaging (Rs-fMRI), Acute ischemic stroke, the International Standard Scalp Acupuncture, amplitude of low-frequency fluctuations (ALFF), the regional homogeneity (ReHo), thevoxel-mirror homotopy connection (VMHC)

Posted Date: November 18th, 2020 
DOl: https://doi.org/10.21203/rs.3.rs-104143/v1

License: (c) (1) This work is licensed under a Creative Commons Attribution 4.0 International License. Read Full License 


\section{Abstract}

Objective: Resting State Functional Magnetic Resonance Imaging (Rs-fMRI) was used to explore the changes on the functional connections between cerebral hemispheres and local functional activities in patients with acute ischemic stroke dysfunction treated by International Standard Scalp Acupuncture.

Methods: 30 patients of acute ischemic stroke in the middle cerebral artery supply area of the dominant hemisphere were selected and randomly divided into treatment group and control group at a ratio of 1:1 according to the random number table method, with 15 patients in each group. Patients in the control group were treated with conventional western medicine, while patients in the treatment group combined the International Standard Scalp Acupuncture (MS5, MS6 and MS7). All the patients were evaluated for efficacy and received whole brain $\mathrm{fMRI}$ scan before and after the treatment. The observation indicators included (1) NHISS score and its sub-item scores related to dyskinesia before and after treatment. (2) the amplitude of low-frequency fluctuations (ALFF), regional homogeneity (ReHo), and voxel-mirror homotopy connection (VMHC) analyzed based on Matlab 2012a platform, SPM12, and REST1.8 before and after treatment.

Results: 1 . The difference of NHISS scores between the two groups was statistically significant before and after treatment $(P<0.05)$, indicates that patients in the treatment group were improved better. 2 . The changes in brain area activation between the two groups of patients were as follows: (1) The values of VMHC increased in brain regions dominated by bilateral BA6 and BA8; (2) The values of ALFF increased mainly in the left BA39 and the adjacent superior temporal gyrus and middle temporal gyrus . (3) The values of ReHo increased in the brain region of left middle temporal gyrus (including BA21) and its extending to BA37, and the left BA40 and angular gyrus and its extending to BA7.

Conclusion: Scalp acupuncture can help the recovery of the patients with acute infarction of superior middle cerebral artery and its mechanism possibly related to the special changes in the regional functional activities of sensory integration, language processing and motor coordination, also the bilateral motor control of frontal lobe.

Trial registration: Trial Registration Number: Chinese Clinical Trial Registry ChiCTR-IOR-15007672. Registration date: November 7, 2015.

\section{Full Text}

This preprint is available for download as a PDF.

\section{Figures}




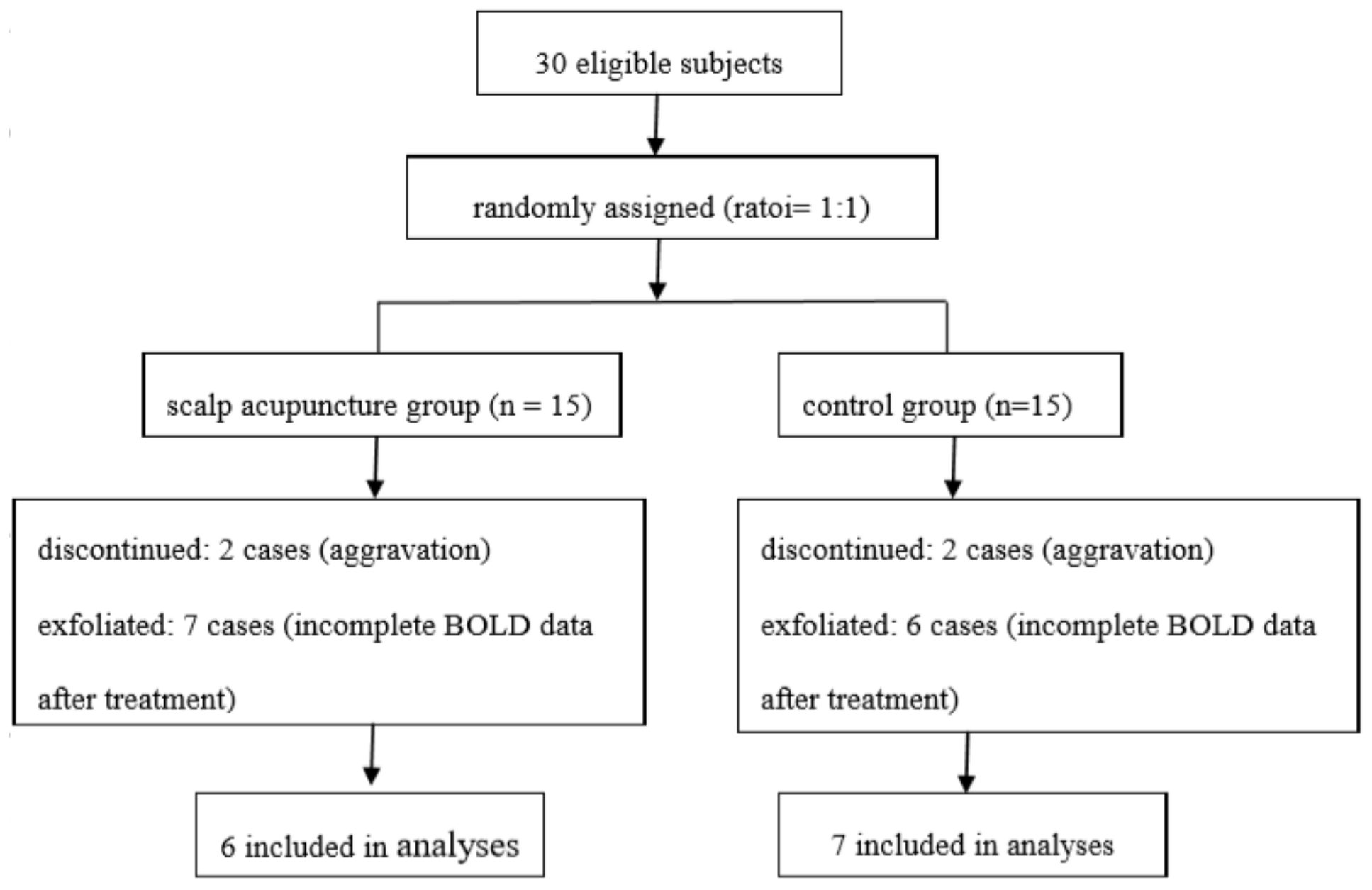

\section{Figure 1}

The Inclusion situation of patients 


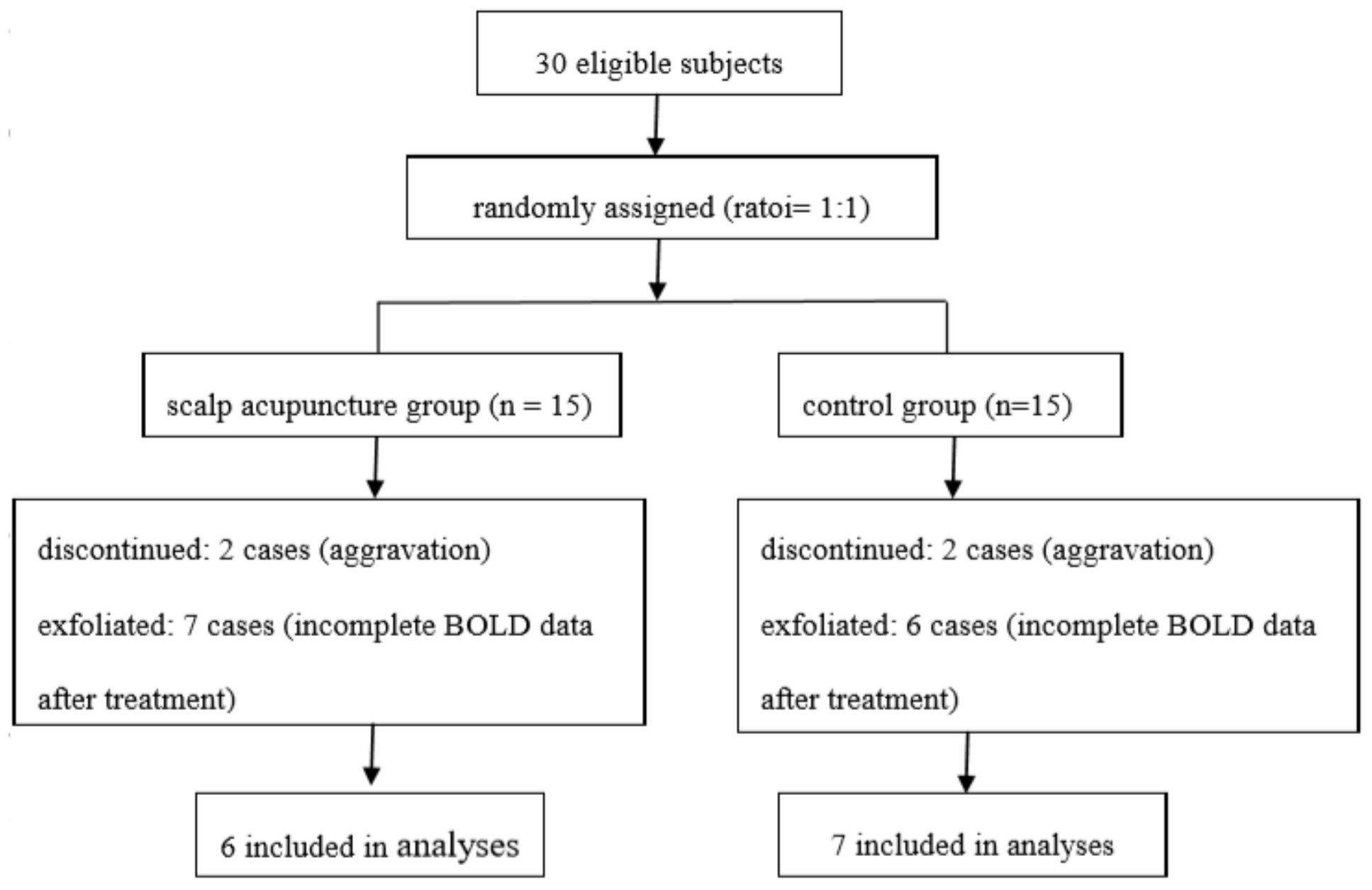

\section{Figure 1}

The Inclusion situation of patients 


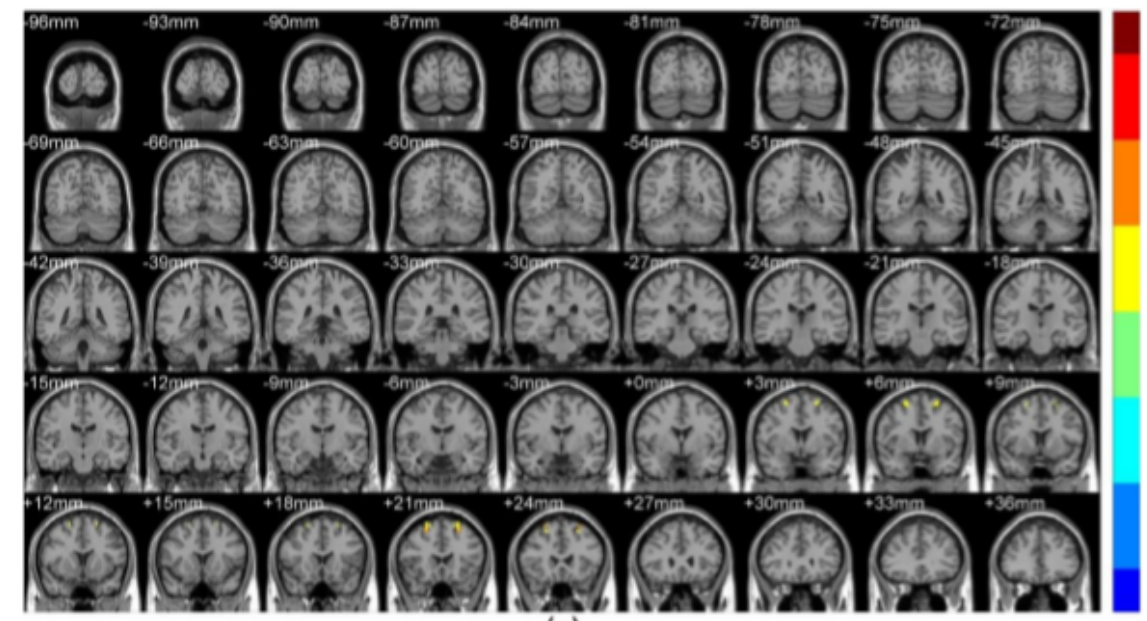

(a)

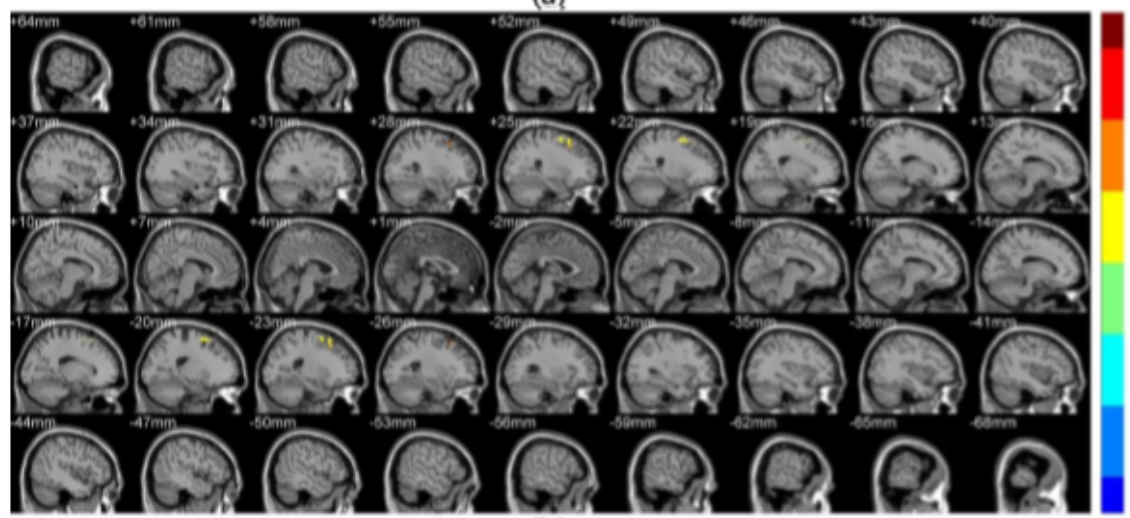

(b)

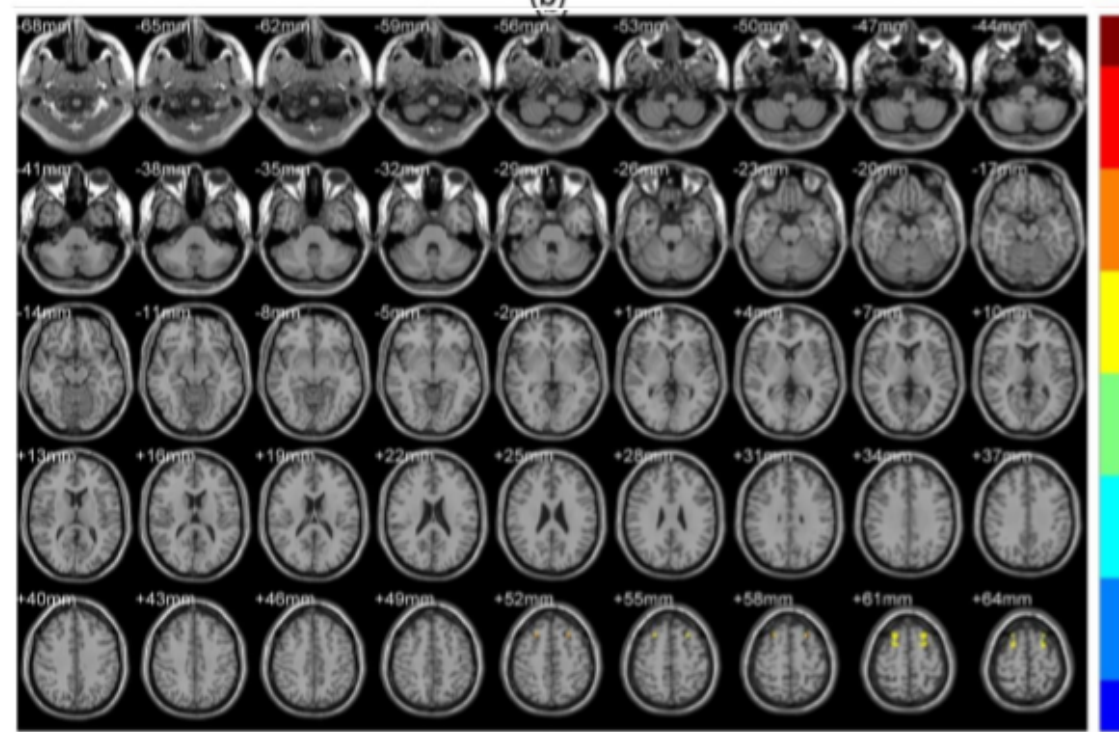

(c)

Figure 2

Brain regions with VMHC specific changes in patients with international standard scalp acupuncture 


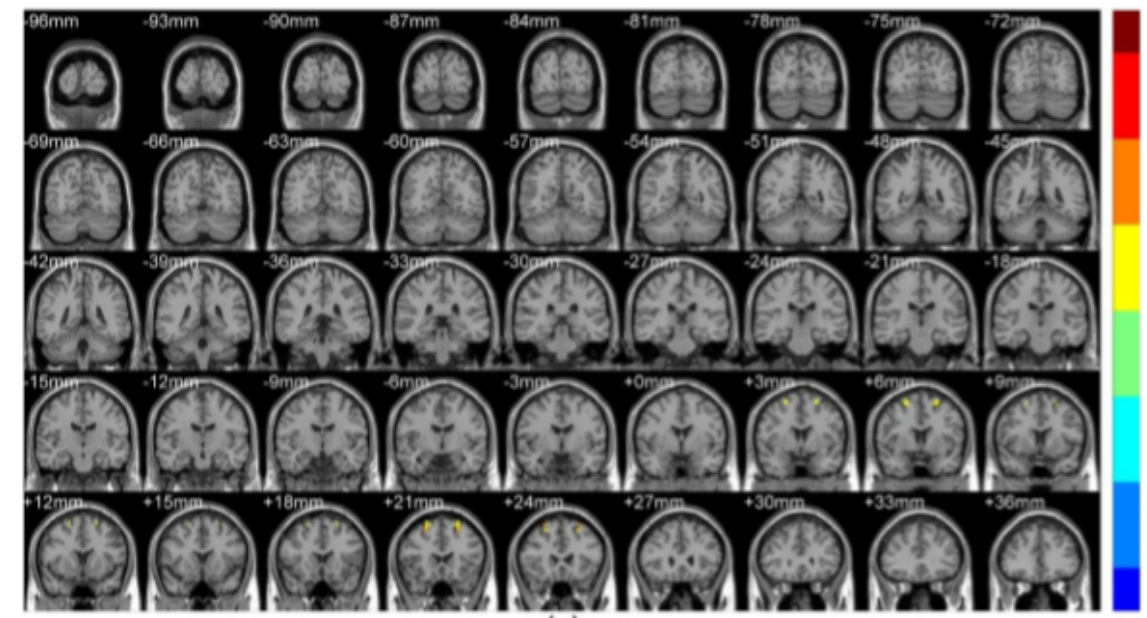

(a)

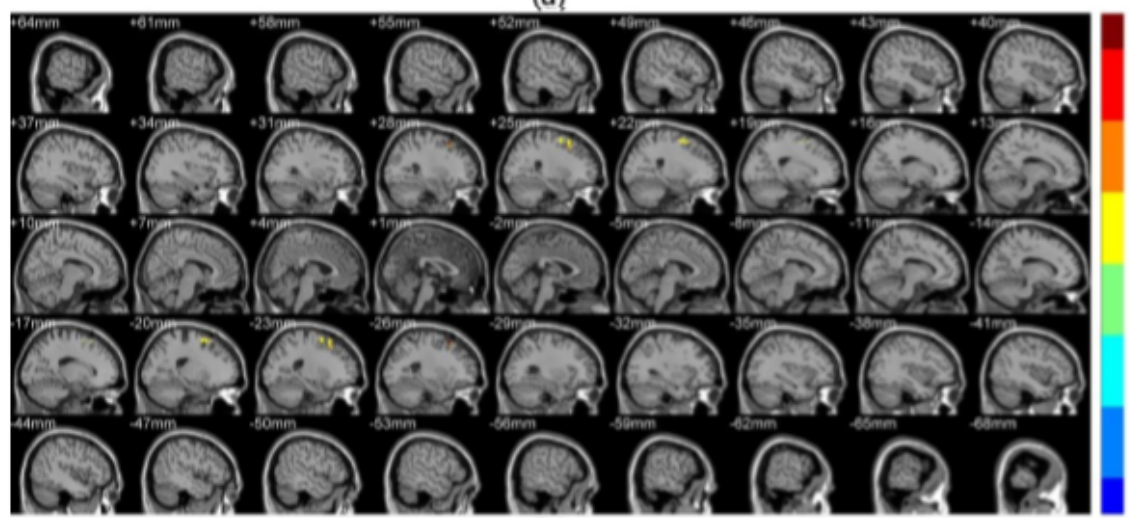

(b)

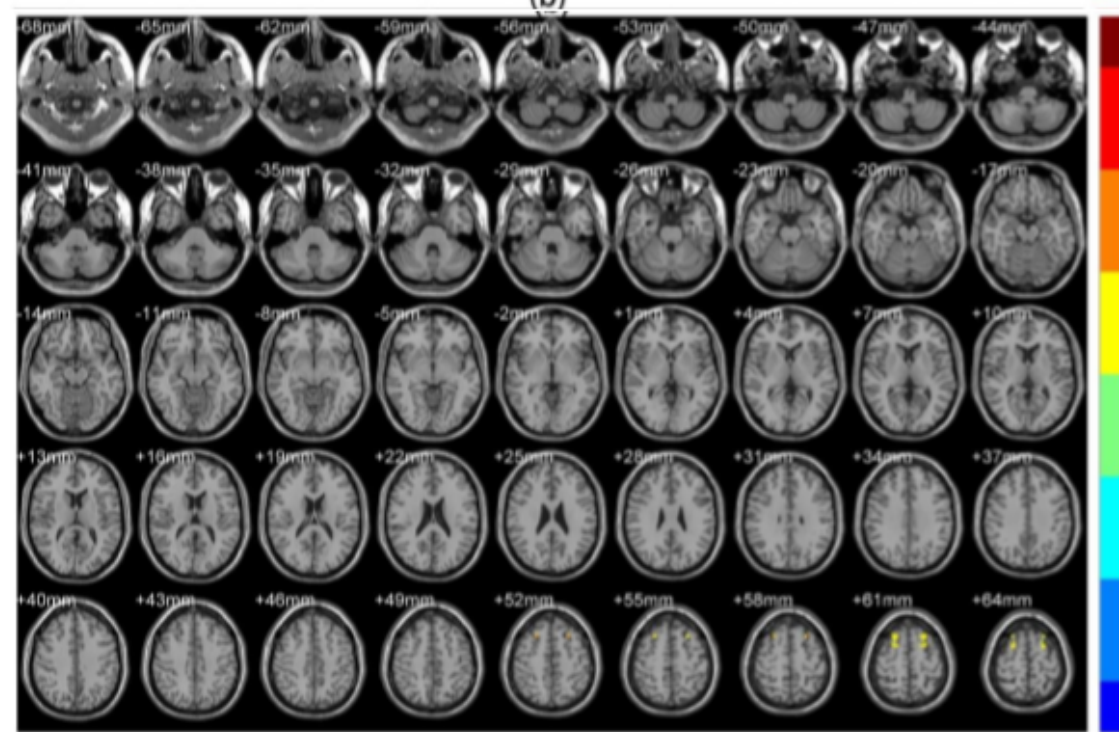

(c)

Figure 2

Brain regions with VMHC specific changes in patients with international standard scalp acupuncture 


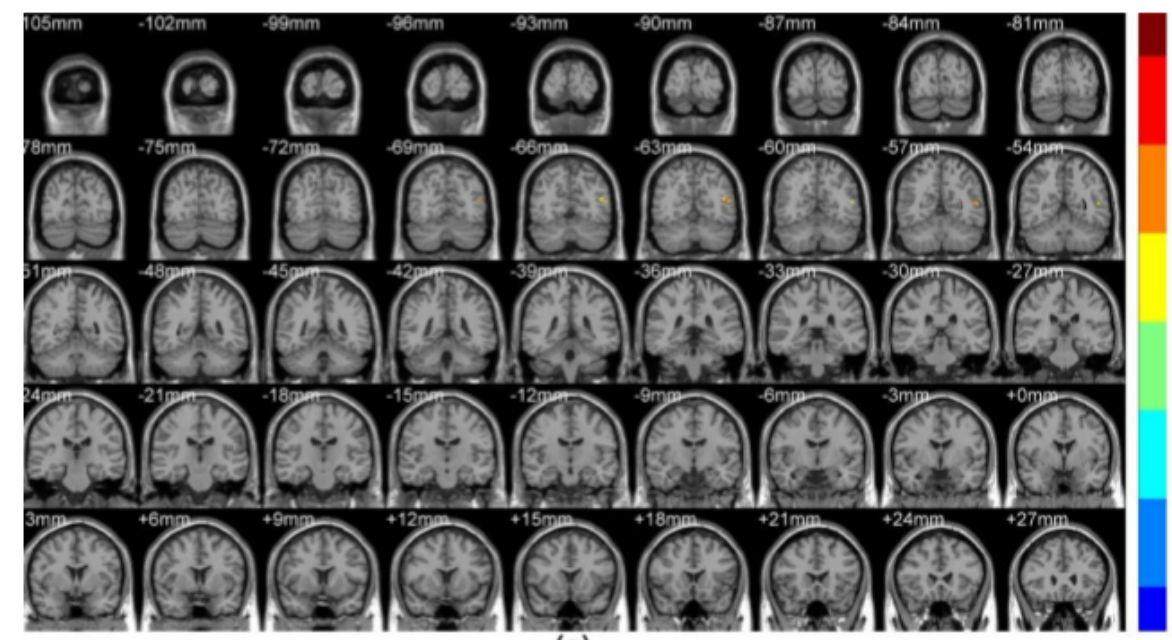

(a)
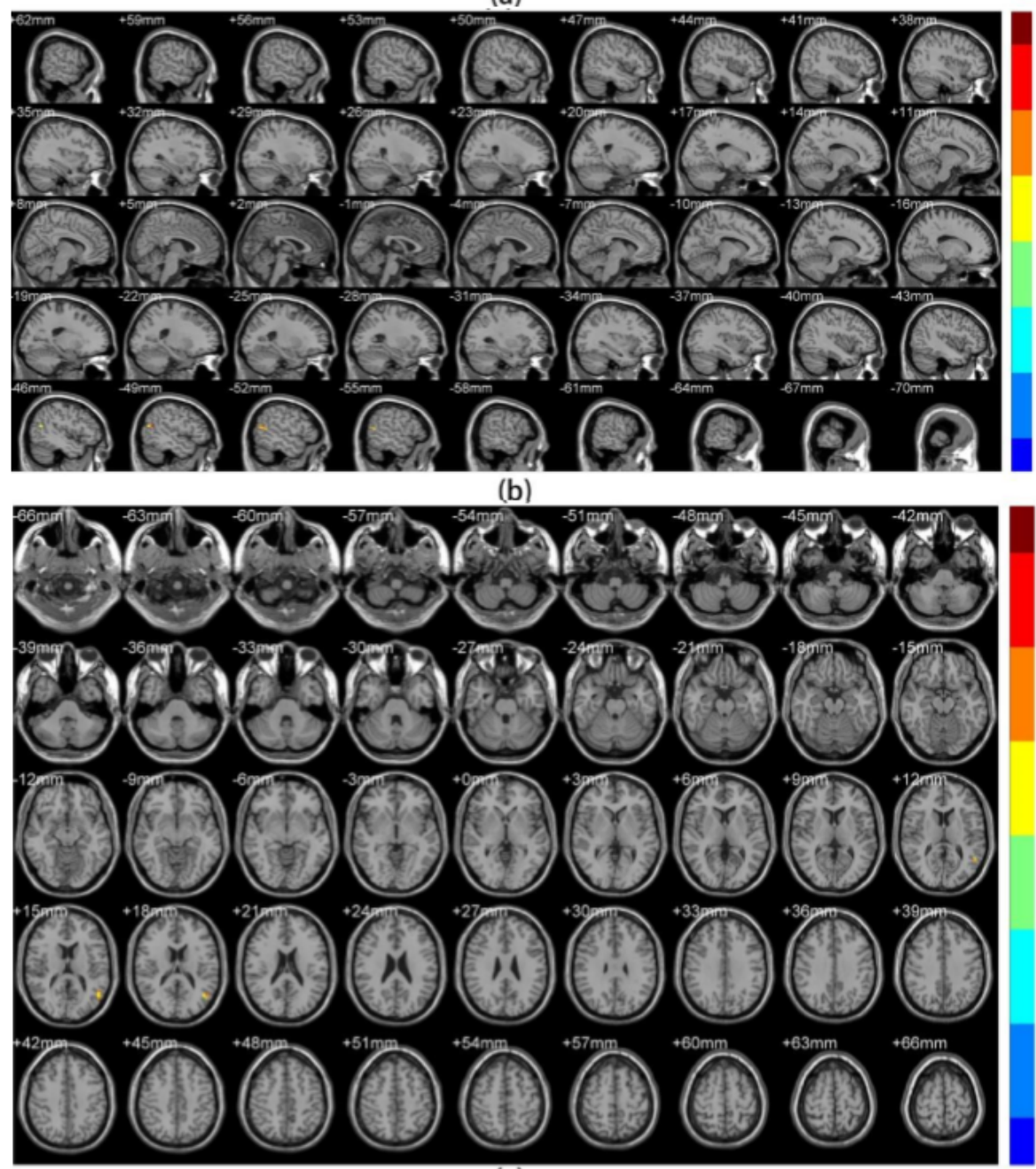

(c)

Figure 3

Brain regions with ALFF specific changes in patients with international standard scalp acupuncture 


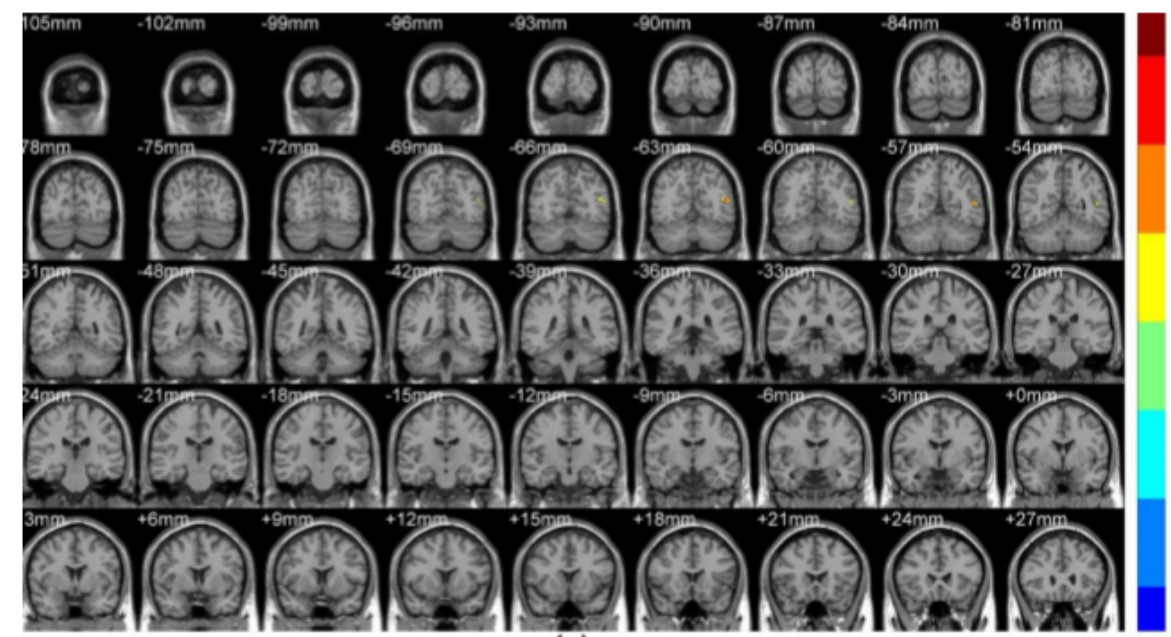

(a)
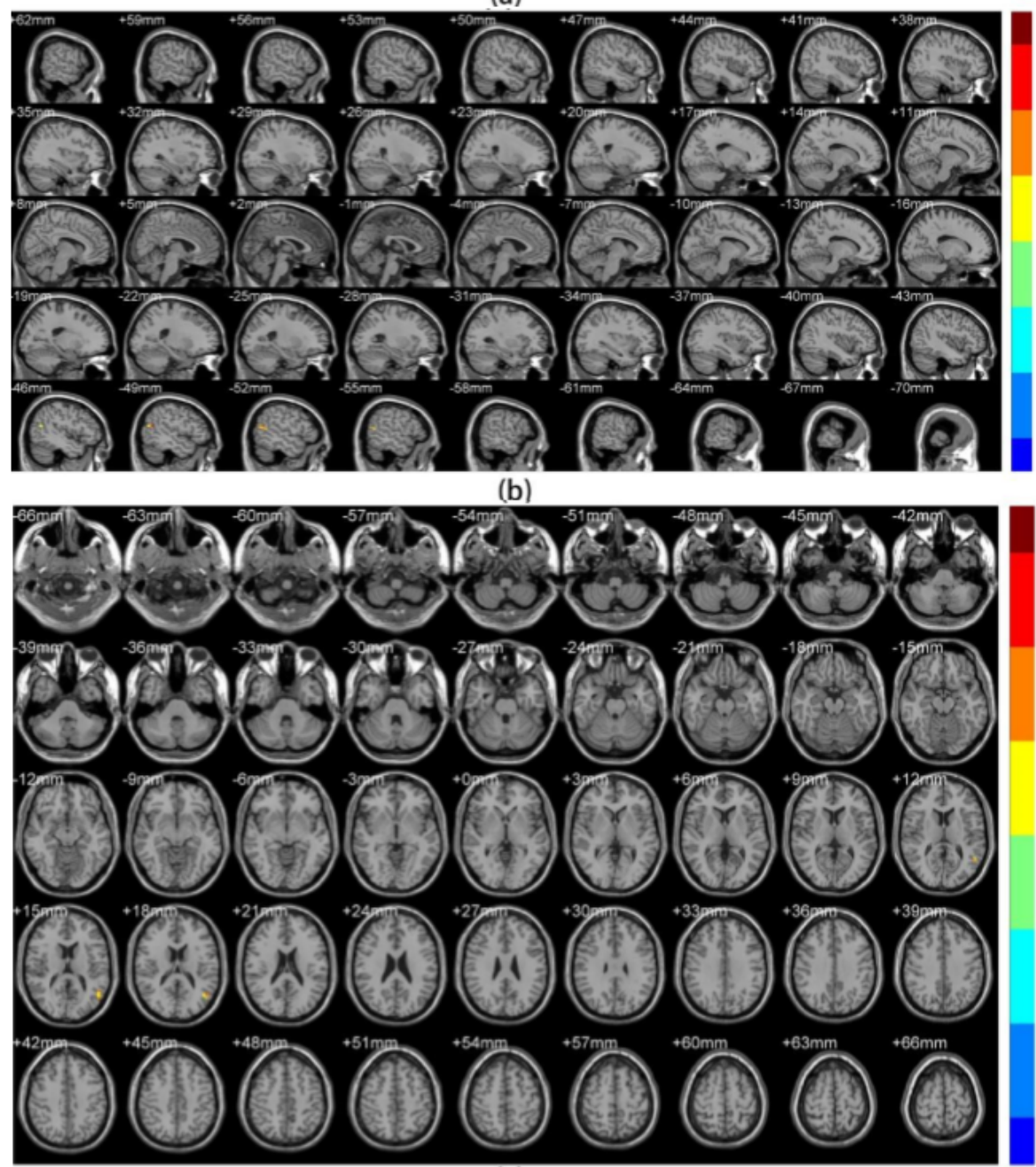

(c)

Figure 3

Brain regions with ALFF specific changes in patients with international standard scalp acupuncture 

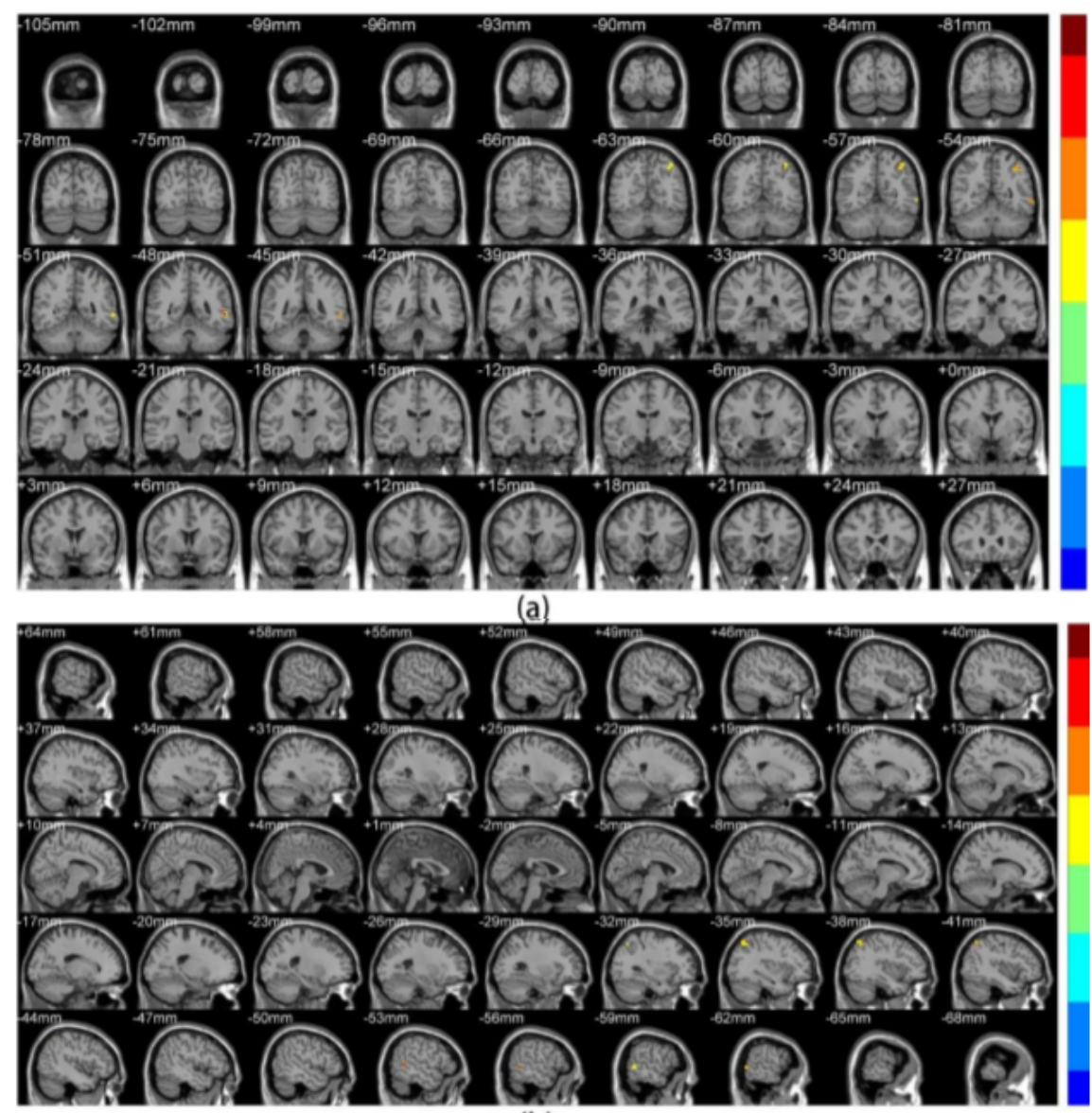

(b)

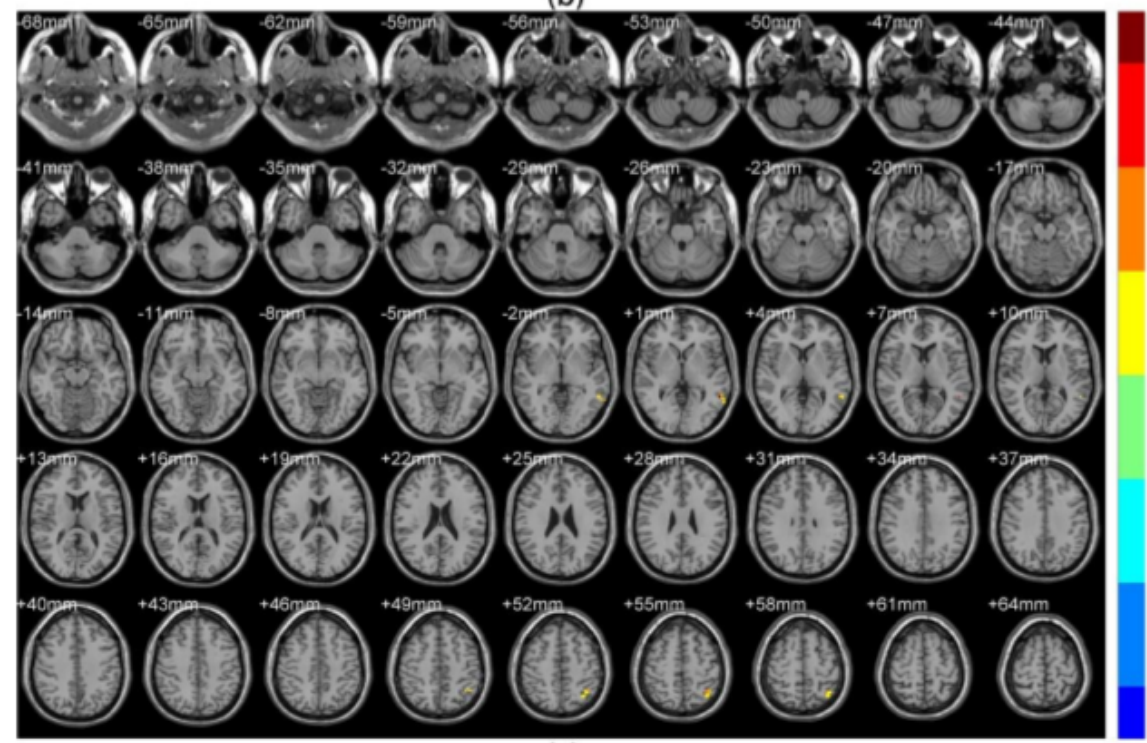

(c)

Figure 4

Brain regions with ReHo specific changes in patients with international standard scalp acupuncture 

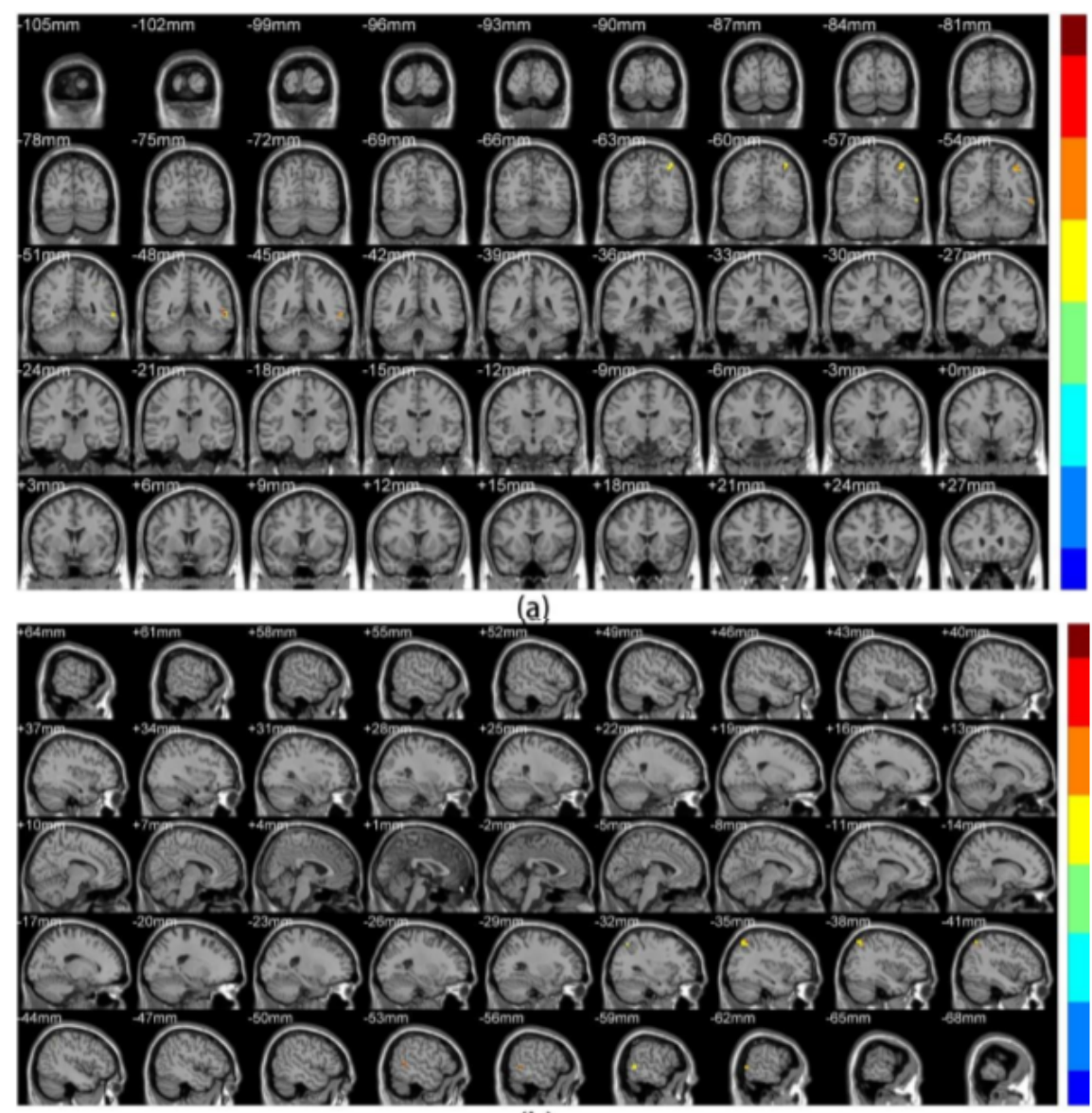

(b)

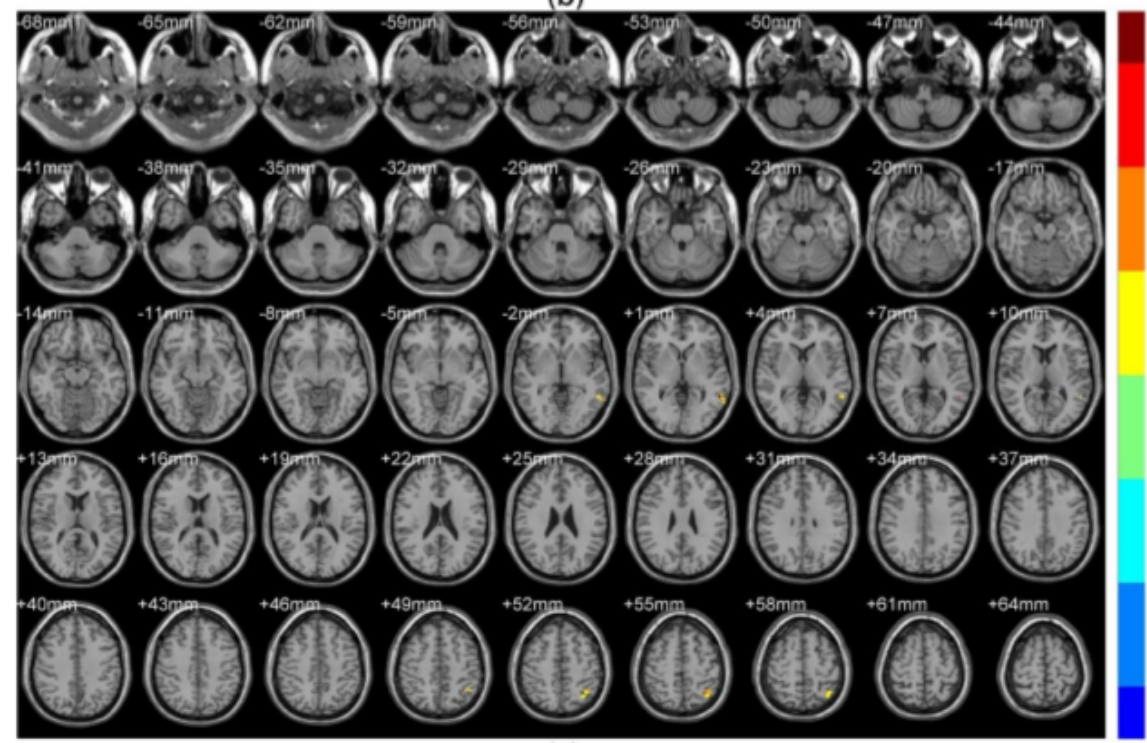

(c)

Figure 4

Brain regions with ReHo specific changes in patients with international standard scalp acupuncture 